\title{
Traduire
}

Revue française de la traduction

227 | 2012

Éco, socio, philo... \& co

\section{La face cachée de la révision}

\section{Charles Martin}

Traducteur : Hélène Ladjadj

\section{CpenEdition}

Journals

\section{Édition électronique}

URL : http://journals.openedition.org/traduire/493

DOI : $10.4000 /$ traduire. 493

ISSN : 2272-9992

Éditeur

Société française des traducteurs

Édition imprimée

Date de publication : 15 décembre 2012

Pagination : 93-100

ISSN : 0395-773X

Référence électronique

Charles Martin, « La face cachée de la révision », Traduire [En ligne], 227 | 2012, mis en ligne le 01 décembre 2014, consulté le 19 avril 2019. URL : http://journals.openedition.org/traduire/493 ; DOI : $10.4000 /$ traduire.493 


\section{La face cachée de la révision}

Conséquence des dernières normes qualité dans le secteur de la traduction, de nombreux fournisseurs de services de traduction imposent révision ou remaniement systématique des textes traduits par un second traducteur, avec l'idée que cela améliore nécessairement la qualité. Mais cette supposée garantie de qualité exige que le second traducteur ait une réelle compétence et que le processus traduction-révision soit effectué correctement. En outre, les améliorations éventuellement apportées par la révision ne justifient pas toujours le temps, les efforts et le coût impliqués. Qui plus est, si la révision n'est pas à la hauteur, elle peut même nuire au résultat final.

\section{Comment garantir la perfection ?}

Bien des lignes ont été écrites sur les qualités et les défauts de la procédure qui consiste à faire vérifier les traductions, face au texte source, par un second traducteur, ce que l'on appelle révision. Néanmoins, l'efficacité de cette procédure pour améliorer la qualité semble rarement mise en doute, pas plus que la nécessité de l'employer systématiquement quel que soit le type de document traduit ou le traducteur.

Si l'on en croit les déclarations affichées sur leurs sites internet : "Toutes les traductions font l'objet d'une relecture par un deuxième traducteur afin de garantir une qualité optimale... ", "Afin d'assurer un travail d'une qualité irréprochable, chaque traduction passe sous une deuxième paire d'yeux ", "La vérification systématique par un deuxième professionnel nous permet de vous garantir la qualité de nos prestations "..., nombre d'agences de traduction sont convaincues que ce système de révision est indispensable. Elles auraient ainsi trouvé la solution permettant d'assurer l'excellence, sinon la perfection : il suffit de faire vérifier le travail du premier traducteur par un deuxième traducteur !

Voilà qui est intéressant. La traduction serait donc devenue la seule profession imposant la vérification systématique du travail par un autre praticien. Voyons voir... Les architectes 


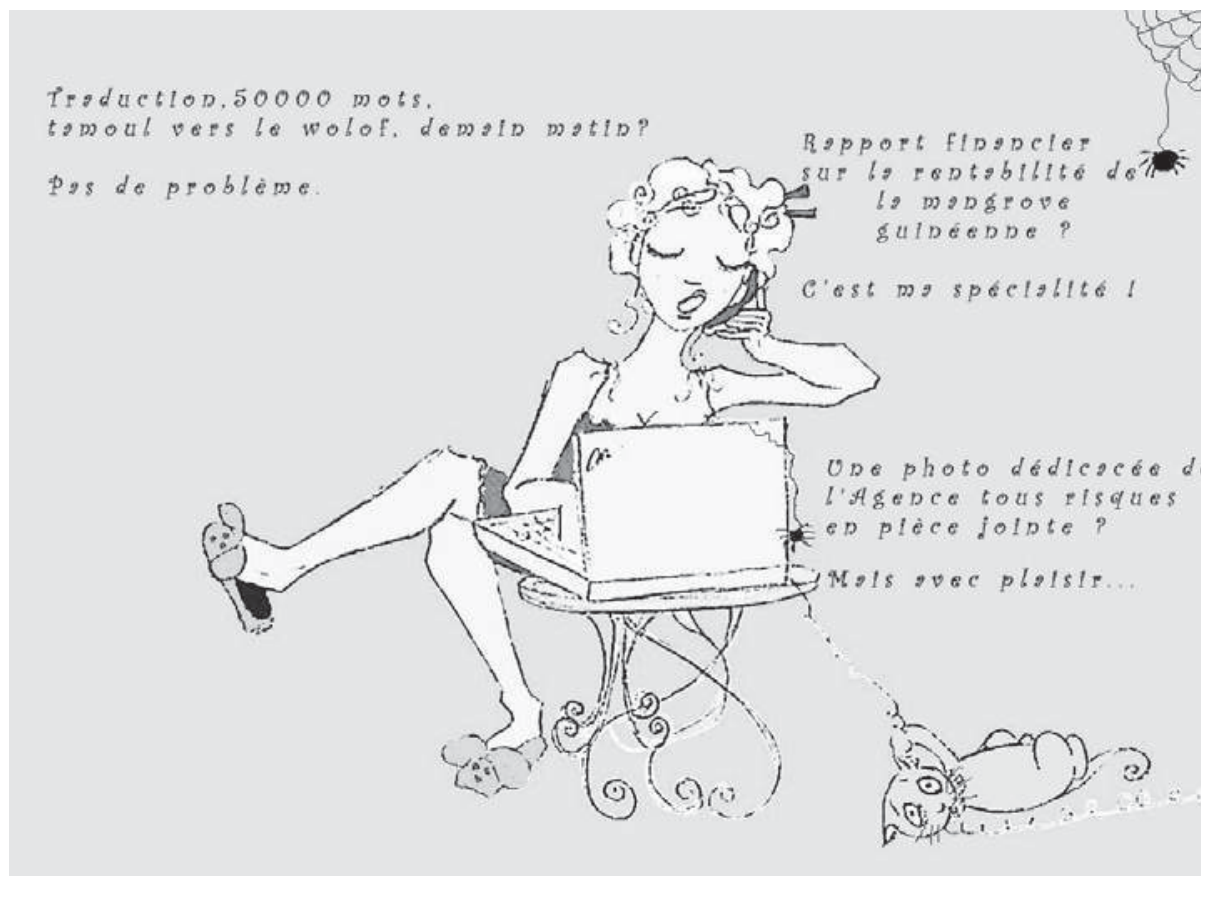

Illustration : Marlène Junius, http://alotoftralala.over-blog.com

peuvent concevoir des bâtiments, les médecins prescrire des traitements et les comptables s'occuper des comptes sans que l'un de leurs collègues vérifie leur travail, mais quand il s'agit de traducteurs, un confrère doit s'assurer qu'ils ont bien travaillé. On peut donc faire confiance aux architectes, aux médecins et aux comptables, mais pas aux traducteurs. Or, si l'on ne peut se fier au premier traducteur pour la qualité d'une traduction, pourquoi le second traducteur, dit réviseur, serait-il plus fiable?

Cette nouvelle méthode d'assurance qualité ne tient pas compte de la nature des documents sensible, complexe ou ordinaire - pourtant décisive, et s'applique à tous les traducteurs, quelle que soit leur expérience ou leur compétence avérée. Cette règle sans nuances met donc tout et tout le monde dans le même sac. Le travail d'un traducteur expérimenté, possédant une parfaite connaissance de la langue source et maîtrisant les domaines dans lesquels il travaille, devra donc être vérifié systématiquement par un tiers, exactement comme celui d'un traducteur novice. Certains traducteurs pourraient mal le prendre, car cela sous-entend deux choses: d'abord, qu'ils n'ont pas une connaissance suffisante de la langue source ou du sujet traité ; ensuite, qu'ils peuvent faire des erreurs par inattention, voire qu'ils ne vérifient pas leur travail.

Cela n'a pas toujours fonctionné ainsi. II était d'usage, dans les organisations internationales, de faire réviser par les traducteurs chevronnés le travail de leurs collègues moins expérimentés, 
tandis que leur propre production n'était pas révisée. Certaines institutions ont sans doute conservé ce type de fonctionnement. Cette nouvelle exigence de l'assurance qualité est liée aux nouvelles normes apparues dans le secteur de la traduction et plus particulièrement les normes EN 15038 en Europe et ASTM F 2575 aux États-Unis. Les fournisseurs de services de traduction (FST) qui appliquent ces normes doivent faire vérifier le texte par une tierce personne qui doit comparer chaque phrase et chaque mot de la traduction avec le texte source afin de vérifier la précision et la qualité générale du travail. En fait, la révision n'est pas obligatoire selon la norme ASTM F 2575 puisque le " demandeur " et le FST peuvent décider d'omettre cette étape. La norme américaine précise toutefois que la traduction " pourrait être de qualité moindre si le processus complet n'est pas appliqué ".

\section{Un peu de terminologie}

La norme EN 15038 dénomme cette opération d'AQ impliquant un second traducteur « révision ", effectuée par un "réviseur ". Lorsque le premier traducteur relit sa traduction face au texte source, EN 15038 parle de "vérification ". Certains nomment ce que fait le second traducteur "révision par un tiers " et ce que fait le premier " auto-révision ". Ce que la norme EN 15038 appelle "révision ", la norme ASTM F 2575 l'appelle " editing " (révision, remaniement). Certains emploient les termes de vérification, relecture croisée, relecture comparée, ou correction, pour désigner exactement la même chose.

Lorsque les mots réviser, vérifier, corriger ou relire sont employés sans référence à une norme qualité, les choses peuvent devenir très ambiguës sachant que cette vérification de la " traduction " peut simplement indiquer une lecture du document traduit sans confrontation avec le texte original, ce que l'on appellera plutôt " relecture ". II s'agit d'une méthode plus simple et moins longue de contrôle qualité qui, appliquée par un lecteur de langue maternelle, peut être très efficace pour détecter les mauvaises traductions et les passages mal écrits pouvant être facilement améliorés. Toutefois, la relecture ne peut vérifier la fidélité au texte de départ et donc la qualité de la traduction. Étant donné la multitude de termes employés, les risques de confusion, d'erreur et de malhonnêteté sont légion. Ici, le terme "révision par un tiers " renvoie à la vérification d'une traduction par rapport au texte source par une personne autre que le traducteur. Lorsqu'il s'agit d'un traducteur vérifiant ainsi son propre travail, on parlera d'« auto-révision ".

\section{Objectif de cet article}

Nous ne prétendons nullement que la révision par un tiers soit inutile. Pratiquée correctement, c'est certainement une façon efficace de former les traducteurs débutants et aussi de vérifier 
les compétences - connaissances, savoir-faire et précision - d'un traducteur. Pas question non plus d'affirmer que cette méthode de révision n'est pas utile à l'assurance qualité : pour certains documents particulièrement importants, elle est certainement souhaitable.

Nous voulons préciser ici que cette méthode de révision n'est efficace pour " garantir " la qualité que si certains principes sont scrupuleusement respectés, ce qui hélas, est rarement le cas, tout particulièrement dans le secteur privé. En outre, s'il y a amélioration de la qualité, cela ne justifie généralement pas le temps passé et les coûts engagés. Pire encore, cette solution peut même nuire à la qualité. Autrement dit, l'opération est souvent superflue et cela peut s'avérer néfaste. En effet, pour la grande majorité des documents, si le traducteur possède une connaissance experte des langues source et cible, maîtrise bien le sujet, dispose d'une solide expérience des techniques et outils de traduction, ainsi que de capacités d'analyse et d'une bonne plume, s'il est consciencieux et pose des questions sur tous les éléments du texte manquant de clarté, alors, faire réviser son travail par un tiers représentera un gaspillage de temps, d'énergie et d'argent.

\section{La révision peut-elle garantir la qualité ?}

Dans quelles conditions la révision peut-elle non seulement améliorer la qualité, mais la " garantir " c'est-à-dire donner l'assurance qu'un document ne contient aucune erreur de traduction et remplit sa mission. Le rôle du réviseur est certes essentiel. II lui faut maîtriser les langues source et cible, bien connaître le sujet traité, posséder une grande expérience des techniques et des outils de traduction, associée à de bonnes capacités d'analyse et d'écriture, être consciencieux et poser des questions sur tout élément du texte nécessitant un éclaircissement. Autrement dit, le second traducteur/réviseur doit posséder lui aussi tout ce dont le traducteur a besoin pour assurer la qualité de son travail : connaissances, capacités et professionnalisme. Sinon, cette méthode de révision ne peut offrir aucune garantie. En effet, deux traducteurs inexpérimentés et/ou médiocres ne sauraient être l'équivalent d'un traducteur compétent et chevronné. Pourtant la norme européenne pour la qualité des services de traduction, EN 15038, n'exige nullement des réviseurs une meilleure connaissance du sujet que celle du traducteur. Elle se contente d'indiquer que les réviseurs devraient posséder une expérience de la traduction dans le domaine concerné. Selon la norme ASTM F 2575, un " editor " n'a nul besoin d'être plus expérimenté ou qualifié qu'un traducteur : il faut simplement qu'il soit " un membre bilingue de l'équipe de traduction...."

Si le réviseur est réellement capable de garantir la qualité, alors le traducteur ne doit pas revenir sur les modifications du réviseur.

Pourtant, on ne saurait ignorer que même si le réviseur est parfaitement compétent, il ne se lance pas dans la révision avec la même connaissance du texte source que le traducteur, sauf 
s'il commence par lire attentivement le document entier, ce qui, a priori, est rarement le cas, notamment dans le secteur privé, pour des raisons évidentes de délais et de coûts. Si l'autorévision et la révision par un tiers supposent, à première vue, la même opération, le traducteur démarre sa révision avec une large avance dans la mesure où il a lu chaque phrase du texte, y a réfléchi et en possède donc une connaissance approfondie. Bref, le traducteur commence le processus de révision avec une meilleure connaissance du document que le réviseur. Le premier contact du traducteur avec le texte source lors du premier jet lui permet en effet de relier les divers éléments, de comprendre ce qui a pu être mal interprété au départ, d'assembler les pièces du puzzle pour assurer la cohérence et supprimer les redondances. L'auto-révision est donc une étape indispensable dans le processus de traduction, et va bien au-delà de la simple vérification qu'il ne manque pas un mot, une phrase ou un signe de ponctuation. Pourtant, chose ahurissante, la norme ASTM F 2575 n'exige pas des traducteurs qu'ils vérifient leur travail ! La norme américaine suppose-t-elle simplement qu'ils le font nécessairement ? Ou se peut-il qu'elle considère que la révision par un autre traducteur rend l'autorévision inutile?

Les réviseurs qui commencent simplement en comparant des paires de phrases l'une après l'autre auront une piètre compréhension du document comparée à celle du traducteur, même s'ils connaissent mieux le sujet. Ils devraient garder cela en tête avant de faire la moindre correction.

\section{Quand la révision peut-elle améliorer la qualité ?}

Si le traducteur initial et son réviseur ne sont pas parfaitement compétents et expérimentés, ni l'un ni l'autre ne pourra garantir la qualité de la traduction. Néanmoins, la révision par un tiers pourra quand même améliorer suffisamment la qualité pour rendre le travail acceptable, notamment si traducteur et réviseur compensent réciproquement leurs défaillances. Mais il est très probable que des erreurs et des ambiguités subsistent et le document traduit ne sera donc pas efficace. Si la révision par un tiers réduit certainement la probabilité d'une très mauvaise traduction, le résultat final peut néanmoins rester bien peu satisfaisant.

Le coût de cette méthode de révision doit aussi être envisagé en termes de rentabilité : les améliorations éventuellement apportées par le réviseur en valaient-elles la peine ? Toute traduction peut toujours être améliorée d'une façon ou d'une autre - rendue plus claire, plus concise, plus lisible. Pourtant, à l'exception des traductions publiées pour lesquelles le style est essentiel, les modifications stylistiques et autres changements mineurs justifient rarement le temps et le coût supplémentaires.

N'oublions pas non plus que lorsque le traducteur et le réviseur ont à peu près le même niveau de compétences, la révision ne sera vraiment efficace que si le traducteur peut valider les choix 
du réviseur, faute de quoi, la compréhension plus superficielle du texte qu'a le réviseur, comme nous l'avons vu plus haut, est susceptible d'introduire des incohérences et des redondances dans la traduction. Si le réviseur ne domine pas le texte ou son sujet aussi bien que le traducteur, le risque existe de le voir en proposer une traduction plus littérale, plus " sûre " mais plus difficile à comprendre pour le lecteur. Enfin, le réviseur peut tout simplement mal interpréter le texte et y introduire des erreurs. Si la traduction révisée n'est pas revue par le traducteur initial pour entériner ou refuser les changements, alors, la traduction pourrait bien ne rien y gagner, à tout le moins. Or, les normes EN 15038 et ASTM F 2575 n'imposent nullement cette étape.

Donc, si le réviseur n'est pas un expert impérativement apte à garantir la qualité, cette procédure de révision ne peut vraiment apporter une amélioration fiable à une traduction que si les changements sont soumis au traducteur afin qu'il valide ceux qui sont justifiés et refuse ceux qu'il estime malvenus. Cette étape est absolument indispensable à une véritable amélioration. Bien sûr, elle alourdit la procédure, allonge les délais et augmente encore le coût de la révision, sans garantie que l'opération soit finalement rentable.

\section{Quand la révision devient néfaste ou nuisible pour la qualité}

Comme nous l'avons vu, une révision négligée ou incompétente qui n'est pas soumise au traducteur peut très facilement nuire à la qualité, jusqu'à rendre le travail inacceptable. Mais la révision peut aussi nuire à la qualité de façon moins évidente.

Supposer que la révision par un tiers est dans tous les cas une assurance qualité ou même une simple amélioration de la traduction, c'est négliger quelques traits fondamentaux de la nature humaine. On peut ainsi se retrouver confronté à divers problèmes, notamment dans le privé, traducteur et réviseur étant souvent en concurrence face à un même client. Un réviseur peut se montrer trop critique et apporter des modifications superflues, sinon néfastes, à la traduction tandis que le traducteur peut refuser des changements pourtant justifiés.

II existe un aspect moins visible mais tout aussi menaçant pour la qualité de la traduction : la dilution des responsabilités. Les normes EN 15038 et ASTM F 2575 ne précisent ni l'une ni l'autre qui, du traducteur ou du réviseur, est responsable de la qualité de la traduction, même si l'on peut supposer qu'elle relève du réviseur, dès lors que le traducteur ne peut valider les modifications. Si la responsabilité n'est pas clairement définie, elle sera alors partagée, de façon implicite et insidieuse.

Bien entendu, le réviseur n'a pu faire sienne la traduction comme l'a fait - ou aurait dû le faire - le traducteur. Même quand le réviseur a le dernier mot, il ne s'agit pas vraiment de sa traduction. Or, étant donné la faible rémunération de la révision dans le secteur privé, même le plus consciencieux des réviseurs ne prendra pas le temps de réécrire tous les passages 
qu'il juge maladroits ou mal dits, surtout si ces changements sont ensuite considérés comme superflus. De ce fait, les réviseurs ne s'impliquent pas totalement dans la traduction concernée. Ce n'est pas "leur bébé ".

Côté traducteur, lorsqu'un réviseur est considéré comme un expert et assume la responsabilité de toutes les modifications, le traducteur peut négliger les recherches et s'en remettre au réviseur pour identifier et remédier à toutes ses défaillances. Cela sera d'autant plus susceptible de se produire si le traducteur est pressé par les délais, comme c'est souvent le cas. Certains traducteurs peuvent aller jusqu'à commettre l'impardonnable en supprimant l'autorévision.

Lorsque le réviseur n'est pas un expert du sujet mais simplement un autre traducteur, une " seconde paire d'yeux ", alors le traducteur comme le réviseur peuvent se reposer à l'excès sur l'autre, en supposant que chacun sache ce qu'il fait. Un traducteur peut se laisser aller aux devinettes pour le sens d'une phrase ou la traduction d'un mot, à charge pour le réviseur de corriger si nécessaire. Ou bien le réviseur éprouvant un doute se laissera convaincre par l'interprétation du traducteur car elle lui semble logique. Rien d'exceptionnel à cela, notamment si le temps est compté.

\section{La révision par un tiers peut-elle être utile ?}

Si le réviseur est un traducteur chevronné qui maîtrise le sujet traité, cette méthode de révision peut s'avérer utile pour l'assurance qualité tout en présentant d'autres avantages. Ainsi, elle permettra de répartir un gros volume entre un traducteur/réviseur expérimenté et un ou plusieurs collègues compétents mais moins expérimentés. Les délais exigeants des clients peuvent ainsi être respectés tout en permettant à des traducteurs moins chevronnés d'acquérir une expérience et un retour précieux dans un domaine précis de traduction.

La révision par un tiers qualifié est en outre le seul moyen de vérifier qu'un document a été correctement traduit et c'est aussi la meilleure façon de tester les compétences d'un traducteur.

\section{Conclusion}

La révision par un tiers garantit la qualité uniquement si le réviseur est un professionnel reconnu, connaissant bien le sujet traité par le document et prenant le temps nécessaire pour comprendre le texte source au lieu de se contenter d'une correction phrase par phrase. Cela n'est guère rassurant car ce n'est pas souvent le cas, surtout dans le secteur privé où la priorité accordée à la rentabilité est rarement favorable à une révision compétente dans la mesure où les tarifs pratiqués par la plupart des sociétés de traduction pour ce service n'incitent pas 
les traducteurs les plus expérimentés, spécialisés, compétents et consciencieux à réviser le travail de leurs collègues. Soyons clairs : pourquoi un réviseur possédant une expertise accepterait-il d'être payé une misère pour corriger le travail de quelqu'un d'autre, alors qu'il peut facturer beaucoup plus en traduisant ?

D'autre part, si un traducteur ne peut pas valider les changements effectués par le réviseur, il est fort possible que la révision n'apporte aucune amélioration et nuise même à la qualité de la traduction. Et si amélioration il y a, la différence en termes de qualité peut être bien trop mince pour justifier le temps et le coût de l'opération.

Même si les normes de qualité ont sacralisé la révision par un tiers comme solution efficace pour l'assurance qualité, il est fort possible que nombre de fournisseurs de services de traduction remettent cette pratique en cause. Certaines sociétés de traduction choisiront certainement une approche plus critique de cette pratique, la réservant aux documents hautement sensibles ou aux situations imposant de confier le travail à des traducteurs moins expérimentés. Quoi qu'il en soit, le marché, par nature, encourage les traducteurs les plus expérimentés et les plus compétents à travailler avec les agences de traduction capables d'apprécier leur talent, de leur faire confiance et de les payer en conséquence.

cmartin@oceanet.fr

Article traduit par Hélène Ladjadj. Cet article est paru dans sa version originale dans Translation Journal en janvier 2012, consultable à l'adresse http://www.bokorlang.com/journal/59editing.htm

Charles Martin a grandi et étudié aux États-Unis avant de venir vivre en France en 1983. Il est traducteur français>anglais depuis 1987. Après avoir travaillé au sein d'une agence de traduction pendant quelques années, il s'est mis à son compte en 1997 et il s'est spécialisé dans la traduction économique et financière. 